\title{
Reliability Analysis of Time Series Force Plate Data of Community Dwelling Older Adults
}

Christoph Bauer ${ }^{a, b, "}$, Ines Gröger ${ }^{a}$, Roland Rupprecht ${ }^{c}$, André Meichtry ${ }^{b}$, Carsten Oliver Tibescu ${ }^{d}$, Karl-Günter Gaßmann ${ }^{a}$

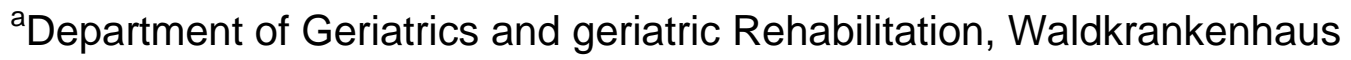
St.Marien gGmbH, Rathsbergerstrasse 57, 91054 Erlangen, Germany,

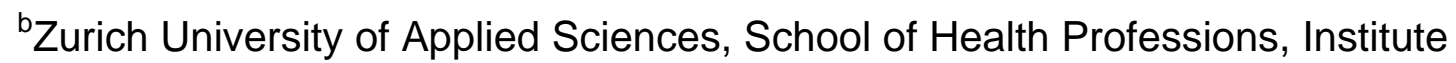
of Physiotherapy, Technikumstrasse 71, P.O. Box, 8401 Winterthur, Switzerland

${ }^{\mathrm{c}}$ Institute of Psychogerontology, Friedrich-Alexander University of ErlangenNürnberg, Nägelsbachstr. 25, 91052 Erlangen, Germany

d Sporthopaedicum Regensburg, Hildegard-von-Bingen-Straße 1, 93053 Regensburg, Germany

"Corresponding author:

Phone: +(41)-934-6449

Fax: +(41)-935-6449

E-mail: Christoph.Bauer@zhaw.ch 


\section{ABSTRACT}

Frequency based analysis of body sway has been used to distinguish between healthy young, healthy elderly adults and elderly adults with Huntingtons disease. Our aim was to assess the reliability of spectral-based outcomes of the Centre of Pressure (CoP) kinematics in order to determine if these outcomes could be tested for their capability to distinguish between elderly fallers and non fallers in a future study. We have studied balance for 30 community dwelling healthy older adults 60 years or older. Four test conditions were used. Three successive trials were performed for each condition. CoP kinematics were estimated with a force platform with three strain gauges set in a triangular position. The frequency content of these signals was estimated. Intrasession Correlation Coefficients (ICC's) were then calculated for all test conditions. The reliability of the selected parameters varied between low and high (ICC 0.652 0.939). The ICC's for the narrow stance tests were higher compared to tests with normal standing conditions (0.771 to 0.94$)$ to (0.652 to 0.865). The highest value was obtained in the high frequency band (0.939). These measures should be viewed with caution when screening geriatric patients because their reliability can not always be assumed.

Keywords: Aged, Falls, Reliability, Posture, Balance

\section{Introduction}

Falls are one of the most common problems facing elderly persons (American Geriatrics Society, 2001). Approximately one third of community dwelling individuals over age 65 fall at least once a year (Tinetti et al., 1988). The number of falls increases with age, to over $50 \%$ for those aged 90 years or older (Tinetti et al., 1988). Impaired balance seems to be an independent risk factor for falls (Corsinovi et al., 2009). Approximately two thirds of elderly people who experience a fall suffer from balance problems (Tinetti et al., 1988). Some of these balance problems are associated with changes of the postural system. Changes in the somatosensory, visual and vestibular system are well known 
(Carter et al., 2001). There is an ongoing search for biomarkers which point to age related changes of the postural system from early onset.

Measurements of the trajectories of the centre of pressure (CoP) in quiet standing on a force platform have been used to characterize the postural control system and changes with aging (Prieto et al., 1996). These measurements can be evaluated using spatial measures (e.g., area of sway or velocity) based on sequential recordings of the CoP on a force platform (Prieto et al., 1996). Alternatively, time and frequency domain measures are based upon the two dimensional time series, compromised of medio-lateral (ML) and anteriorposterior (AP) components of the CoP (Prieto et al., 1996, Myklebust et al., 2009). The ML and AP time series are often analyzed separately or combined into a composite time series (Myklebust et al., 2009). Time series data have been used to distinguish between healthy young, healthy elderly adults and elderly adults with Huntington's disease (Prieto et al., 1996, Amoud et al., 2007, Myklebust et al., 2009). Previous studies indicate that frequency based measures of postural steadiness change significantly in age and disease (Prieto et al., 1993). Time series data of CoP signals have been used to classify elderly persons who are at risk of falling (Williams et al., 1997, Aufauvre et al., 2005) or for diagnosis of selected pathologies, such as primary orthostatic tremor (Karlberg et al., 2003) or unilateral labyrinth disability (Demura and Kitabayashi, 2006). It has been shown that selected bands (AP $0.5-2 \mathrm{~Hz}$ ) can differentiate between fallers and nonfallers (Aufauvre et al., 2005) and that measures of the frequency of CoP movement are sensitive to age related changes between eyes open and eyes closed conditions in elderly adults (Prieto et al., 1996).

If these measures are also suitable for follow up assessments is still discussed. One study showed improvement in functional balance tasks but not in time series force plate measurements in elderly nursing home residents which attended a balance training program (Rugelj, in press).

Previous studies have shown that reliability of static posturographic measurements can not always be assumed (Doyle et al., 2005, Bauer, 2008a,). Before a test protocol is used to differentiate between fallers and non fallers its reliability should be assessed first. In a preceding study we assessed the reliability of a test protocol, using spatial measures of the CoP (Bauer et al., 2008b). The presented study uses time series data, obtained with the same test 
protocol and the same force plate. The aim of this study was to assess if the presented instrument is suitable to differentiate between subjects. The results shall be used in a future study to differentiate between fallers and non fallers.

Parameters calculated from the CoP signal are influenced by, among others the position of the feet (Bauer, 2008a) and vision (Prieto et al., 1996). Melzer et al found an increase in medio-lateral sway in narrow base stance in older people who experienced recurrent falls (Melzer et al., 2004). Consequently it is interesting for clinicians and researchers alike to investigate on the response of an elderly person's postural system to different, more challenging standing positions. For that reason we present a paper discussing the reliability of time series data measured with different foot placements and eyes open and eyes closed, using static posturography, on a population of community dwelling older adults.

\section{Subjects and Methods:}

\subsection{The Study Sample}

30 (22 women, 8 men) community dwelling elderly persons participated in this study. The subjects were part of a larger sample of community dwelling older adults, participating in an intervention study assessing the effect of group exercises on balance performance (Bauer et al., 2009). The subjects balance spectrum at baseline, prior to this study, was estimated with the Tinetti Balance Score, the Berg Balance Scale, the Five Chair Rise Test and the Timed Up and Go Test. Individuals were considered community dwelling if they lived independently in their own home. They were recruited through a newspaper advertisement and informative meetings. The sample consequently was selfselected. Criteria for inclusion in this study were as follows: Participants had to be 60 years old or more; had to be able to walk 10 meters with or without a walking aid; had to be able to stand independently for 90 seconds; and had to be able to understand and follow verbal instructions. Exclusion criteria were: Acute or sub acute diseases of the cardiovascular or the respiratory system and/or severe cognitive impairment. Cognitive impairment was quantified with the Mini Mental State Assessment (MMS). The Ethics Committee of the 
Friedrich-Alexander-University-Erlangen-Nürnberg approved the study. All subjects signed informed consent paperwork prior to participation in the study. The presented study complies with the declaration of Helsinki and it's amendments from Tokyo, Venice and Hong Kong.

\subsection{Apparatus}

$A P$ and $M L$ CoP trajectories were estimated with a SATEL ${ }^{2}$ force platform with three strain gauges set in a triangular position. The platform measures $480 \times 480 \times 65 \mathrm{~mm}$ and weights $12 \mathrm{~kg}$. The sensitivity of each sensor is certified to be $0.0017 \%$ for a maximum load of $100 \mathrm{~kg}$ per sensor. A variation of $9.8061 \mathrm{~N}$ of the force applied to one strain gauge corresponds to a $50 \mathrm{mV}$ variation of the output. The output range runs from 0 to $5 \mathrm{~V}$. The system uses a 12 bit analogue-digital converter and a rejecter filter of $50 \mathrm{~Hz}$ before applying the analog-digital converter. Force data were sampled at $40 \mathrm{~Hz}$. The electronic filter on the board is dimensioned to cut the power supply interferences (over $50 \mathrm{~Hz}$ ). The 0 - 0.5 component does not include a DC term. No trend removal was carried out. The signal is moved by $M L$ mean, respectively AP mean to become a zero mean signal. The digital data were transferred via a USB port to a personal computer by SATEL ${ }^{a}$ software. Centre of pressure (COP) trajectories were assessed in the AP and $\mathrm{ML}$ direction. Using Fast Fourier Transformations (Cooley Tokey Algorythm) (FFT) the frequency content of these signals was estimated. The integral of this signal is calculated as the area under the power curve, "as power density". The frequency vector was categorized into three bands (1: $0-0.5 \mathrm{~Hz} ; 2: 0.5-2 \mathrm{~Hz} ; 3:>2 \mathrm{~Hz}$ ) in order to calculate the energy content within these frequency bands.

\subsection{Procedure}

The test conditions (light, room temperature) were standardized before the tests. The same two experienced researchers conducted all trials. The individuals stood quietly on the force plate for three successive trials with two minutes rest between trials. There was no practicing trial before the measurements. The visual environment was stable. There was constant, 
indirect light and the reference point did not move. The individuals maintained each test position for 30 seconds. Data were recorded during the last 25.6 seconds. All tests were done with shoes removed. The individuals were instructed to look straight ahead with their head erect, their arms resting at their side, while maintaining balance. Each subject was instructed by one examiner: "I want you to stand upright, with your arms resting at your sides, without moving or speaking until you get further instructions." Each trial consisted of four tests. The test conditions were: 1$)$ normal standing $(2 \mathrm{~cm}$ heel distance and $30^{\circ}$ angle between feet) with eyes open (EO), 2) normal standing with eyes closed (EC), 3) narrow stance with eyes open (NEC) and 4) narrow stance with eyes closed (NEO). The narrow stance position was determined by using a vertical red line in the middle of the force plate. Three successive trials were preformed for each condition.

\subsection{Statistical analysis}

All calculations were done using SPSS 14.0 for Windows. The data were tested for normal distribution using the Kologmorov-Smirnov test. We estimated the reliability of the parameters using Intraclass correlation coefficients (ICC). The ICC Two-Way Mixed Effect Model (Absolute Agreement Definition) described by Shrout and Fleiss (Shrout and Fleiss, 1979) was chosen because it considers random effects over time. For each ICC the 99\% confidence interval $(99 \% \mathrm{Cl})$ was calculated to consider sampling variations. The ICC was calculated as follows,

$$
I C C=M S_{b}-M S_{E} /\left\{\left[M S_{b}+(n-1) M S_{E}+n\left(M S_{R}-M S_{E}\right)\right] / k\right\}
$$

where $\mathrm{MS}_{\mathrm{b}}, \mathrm{MS}_{\mathrm{r}}$ and $M S_{\mathrm{E}}$ are the mean squares of the two-way ANOVA; " $\mathrm{k}$ " is the number of trials and " $n$ " is the number of subjects. According to Walter et al (Walter et al., 1998) 30 subjects and three trials allow ICC estimations of .95 with a type 1 error of .05 and a type 2 error of $.2, \mathrm{HO}$ being $\mathrm{r}=0$. Interpretation for this study's results used the scale proposed by Portney and Watkins (Portney and Watkins, 2000) that defines 0.75 as the lower value for moderate agreement and 0.9 as the lower value for high agreement. A 99\% CI for the ICC indicates the likely range of values containing the true population. A wide $99 \%$ 
$\mathrm{Cl}$ suggests poor precision, and a narrow 99\% Cl suggests good precision. The coefficient of variation (CV) was calculated as follows,

$$
C V_{p, i}=S D_{p, l} / p_{\text {mean }}
$$

where $S D_{p, i}$ is the standard deviation and $p_{\text {mean }}$ is the mean for the variable $p$ for each subject i. The overall CV was calculated as the mean $\mathrm{CV}$ for each variable p. To contrast the variability of the measurements Fmax tests were conducted.

\section{Results}

\subsection{Sample characteristics}

All subjects were able to complete the tests. No dropouts were recorded. Participants' characteristics are presented in Table 1. The original sample showed a mean Tinetti Test score of $24.84( \pm 2.95)$, a mean Berg score of 49.9 $( \pm 5.53)$, a mean repeated chair stands test of $14.55( \pm 4.91)$ and a mean Timed Up and Go Score of $9.63( \pm 3.39)$. The mean MMS score was $29.41( \pm 0.96)$ (Bauer et al., 2009).

\subsection{Descriptive Statistics}

ML signal: The descriptive analysis revealed that the main power content of the ML signal is $0-0.5 \mathrm{HZ}$. Body sway increases in the narrow stance conditions (NEO and NEC) compared to normal standing conditions (EO and EC). In the NEC position body sway was highest, compared to all other positions, in all frequency domains. The data shows very large standard deviations and variability.

AP signal: The descriptive analysis revealed that the main power content of the AP signal is $0-0.5 \mathrm{HZ}$. Body sway increases under eyes closed conditions (EC and NEC), compared to eyes open conditions (EO and NEO), unlike body sway in the ML direction. In the NEC position body sway was highest, compared to all other positions, in all bands. The data shows very large standard deviations and variability. The descriptive data is shown in Tables 2 and 3. 


\subsection{Tests for normal distribution}

The Kologmorov Smirnov tests revealed that our measures were not normally distributed.

\subsection{Reliability}

Reliability data are provided in Table 4 and Table 5. The energy content outcomes showed low to high reliability. The reliability was better during narrow stance conditions (NEO and NEC) compared to EO and EC. NEC showed the best reliability in both directions while EO showed the worst. Band 1 showed to be most reliable during NEC in the ML direction and during NEO in the AP direction. Bands 2 and 3 are more reliable in narrow stance conditions (NEO and NEC), compared to EO and EC, in both directions. For both bands the highest reliability was obtained during the NEC test.

\subsection{Fmax Tests}

Fmax tests showed reliable differences in variability across all four tests. The results are presented in Table 6.

\section{Discussion}

The Intrasession reliability of energy content of a CoP signal was assesed with an ANOVA based ICC model and the CV. ICC's indicate the potential to discriminate between participants giving an idea of the diagnostic value of a measure (e.g. between subjects designs) while CV's show the capacity to detect changes over time (e.g. the effect of a rehabilitation program, within subjects designs). We found that reliability was higher in narrow stance conditions, compared to normal stance. We further found that reliability increased when subjects were asked to close their eyes and to close their eyes while maintaining the narrow stance position. The reliability of the selected parameters varied between week and excellent (ICC $0.652-0.939$ ). To test 
homogeneity of variance, Fmax tests were conducted. Fmax tests showed reliable differences in variability across all tests. This indicates the influence of the test position on the parameters. The $99 \%$ Cls were examined for overlap in order to assess statistically reliable differences between the ICC's.

In the $\mathrm{ML}$ direction the $99 \% \mathrm{Cl}$ of the ICC for the complete signal and for band 2 during the NEC condition are higher, compared to all other measurements of ML direction. The authors therefore recommend the NEC test for measures in the ML direction.

In the AP direction the $99 \% \mathrm{Cl}$ of the ICC for band 2 NEC is an outlier, being more narrow and higher, compared to all other parameters of $A P$ measurement. This indicates very good repeatability. Other outliers are the $99 \% \mathrm{Cl}$ 's for EO ML direction, all frequency bands except band 2, where the lower margin is especially low, compared to the other $99 \% \mathrm{Cl}$ 's. We therefore recommend excluding these measures in future research and assessment. We authors recommend the NEC test for measures in the AP direction. To achieve more reliable measures it is recommended to use narrow stance conditions such as NEO or NEC. Independent of the direction and the band, narrow stance conditions yielded better results than normal stance conditions.

The CV's ranged from $19 \%$ to $34 \%$. The study of Rogind et al has shown lower CV's of posturographic measurements, but differs in terms of measurement protocol; population and test design (Rogind et al., 2003).

Previous studies indicate that frequency-based measures of postural steadiness change significantly in age and disease (Prieto et al., 1993, Myklebust et al., 2009) .The observed sway in quiet standing represents the output of the complex sensorimotor system of integrated information from the visual, vestibular and somatosensory system (Myklebust et al., 1995). The CoP time series may represent the dynamics of a nonlinear system (Johansson and Magnusson, 1991). While up to date the presented measurement does not allow for a diagnosis of defined disorders, with exceptions (Karlberg et al., 2005, Demura and Kitabayashi, 2006), it records abnormalities in the sensorymotorsystem, on a deeper level than a clinical assessment. Postural dyscontrol in the elderly may reflect sub clinical pathologies affecting one or more components of the postural control system, as well as age related changes in the sensorimotor systems (Horak et al., 1989). These sub clinical 
changes can be detected using time series data of CoP kinematics. The presented alterations to the subjects standing position allow the clinician to investigate the capability of the subject to adapt to new, more challenging positions and to record the responds of the system to the changes in detail. When screening patients for balance disorders, it appears to be interesting to assess the reliability of these high frequency components of body sway. This protocol enables the clinician to discover sway frequencies in the high frequency domain which is not possible with classical balance tests, gait assessment or other posturographic tests. The authors recommend using this instrument together with other clinical tests and gait analysis.

In narrow stance control of lateral stability (ML trajectories) appears to be important and loss of ML control is associated with falling (Melzer et al., 2004). Melzer et al., using spatial measures of CoP trajectories, found an increase in ML trajectories in narrow base stance in older people who experienced recurrent falls (Melzer et al., 2004). Fallers in the study by Maki et al. showed increased ML trajectories and lateral spontaneous sway was the single best predictor of future falling (Maki et al., 1994). However other studies found no association between force platform measurements and falls (Piirtola and Era, 2006). We assessed the reliability of selected parameters because poor reliability might be one explanation why some studies found no association between posturography and falls. One proposition for future research is to test if time series data of CoP trajectories can differentiate between fallers and non fallers.

Compared to other protocols of static posturography the presented reliability coefficients are moderate to good (Bauer, 2008a). Other studies regarding the reliability differ in terms of device used, participants, measurement protocol and parameters (Bauer, 2008a). Because of methodological differences and the different results regarding the validity of force platform measures, we think that each protocol of posturography has to be evaluated for its reliability. The next step will be to analyze the validity of the presented protocol in an ongoing study.

The results might be influenced by the variability of the data. ICC's are highly influenced by variability and easier conditions often have lower ICC's due to lower variability. The heterogeneity of our participant sample (both genders, 
varying level of balance) could increase the variability among them and consequently lead to higher reliabilities.

One or more practice trials might improve the reliability of the measurements. To avoid fatigue of the elderly test subjects this was not done.

The choice of the exclusion criteria's might have affected the results. The authors did not exclude conditions like arthritis or neurological conditions because a wide range of medical conditions and symptoms related to balance and gait abnormalities are common in geriatric patients (Corsinovi et al., 2009, Gaßmann et al., 2009). Our patients therefore represent the population which is most likely to receive this test. We recommend repeating this study on certain subgroups of patients. Furthermore differences between genders should be assessed as posturographic measures show differences between genders (Masui et al., 2005).

The choice of the frequency components might have affected the results. The selected frequency components were chosen according to the literature. The first band $(0-0.5 \mathrm{~Hz})$ was chosen because of general agreement among other researchers (Soames and Atha, 1982), that a peak of the body's oscillations occurs in this frequency domain. The intermediate band $(0.5-2 \mathrm{~Hz})$ was chosen due to the findings of Njiokiktjien and Folkerst (Njiokiktjien and Folkerts, 1971), who state that a second peak occurs in this domain. The high frequency band $(>2 \mathrm{~Hz}$ ) was chosen because certain pathologies might manifest in higher domains (Karlberg et al., 2005). The international standard (Kapteyn et al., 1983) recommends a high frequency domain of $>2 \mathrm{~Hz}$. Some authors argue that, while suitable to discover certain pathologies, this is not useful when testing healthy persons (Demura and Kitabayashi, 2006).

When using spectral analysis of a signal, the significance of the measurement is dependent on the measurement period (Carpenter et al., 2001). The factor representing the lowest frequency recorded by spectral analysis is defined as: 1 /measurement period (Carpenter et al., 2001). The lowest recorded frequency of the reported experiment is therefore 1/25.2 seconds $=0.039 \mathrm{~Hz}$. Using longer measurement periods would have resulted in recordings of slower frequencies. CoP movements show frequencies which can only be recorded after more than one minute of measurement (Carpenter et al., 2001). The authors chose short test duration in order to avoid fatigue of the 
elderly test subjects. The subjects were given no practice trials. One or more practice trials might improve the reliability of the measurements. To avoid fatigue this was not done.

Further applications for the presented analysis in geriatric medicine, screening and training of persons with balance problems are subject to future research.

\section{Conclusion}

This study established the reliability of time series data of postural sway, across four test conditions. Five variables showed excellent reliability $(>0.9)$. They can be recommended for further use in clinical trials. Eyes closed conditions showed better reliability than eyes open conditions. We recommend using eyes closed conditions when assessing static balance control. Narrow stance conditions showed better reliability than normal standing conditions. We therefore recommend narrow stance conditions when assessing static balance control. The factors influencing these conditions must be assessed in future studies. The test procedure is time-efficient, which makes it suitable for hospital settings and for screening large amounts of patients.

\section{Conflict of Interest Statement}

None

\section{Acknowledgements}

This study was not financially supported by any funding sources.

\section{Suppliers}

a. Satel, Rue du Limousin, Blagnac, France 31700. 


\section{References:}

American Geriatrics Society, British. Geriatrics Society and American Acedemy of Orthopedic Surgeon Panel on Falls Prevention, 2001. Guideline for the Prevention of Falls in Older Persons. JAGS, 49, 664 - 672.

Amoud, H., Abadi, M., Hewson, D.J., Michel-Pellegrino, V., Doussot, M., Duchene, J., 2007. Fractal time series analysis of postural stability in elderly and control subjects. Journal of Neuroengineering and Rehabilitation, 4, 12.

Aufauvre, V., Kemoun, G., Carette, P., Bergeal, E., 2005. Évaluation posturale à domicile chez la personne âgée : comparaison chuteurs-non chuteurs. Annales de Réadaptation et de Médecine Physique, 48, 165-171 (in French).

Bauer, C., 2008a. Reliability of Static Posturography - Review. Physioscience, 4, 65 - 70 (in German).

Bauer, C., Gröger, I., Rupprecht, R., Gassmann, K.G., 2008b. Intrasession reliability of force platform parameters in community-dwelling older adults. Arch Phys Med Rehabil, 89, 1977-82.

Bauer, C., Rietsch, C., Gröger, I., Gassmann, K.G., 2009. Mobility and safety for elderly (MoSi) ${ }^{\circledR}$, a new intervention to improve mobility and gait in elderly people. Zeitschrift für Gerontologie und Geriatrie, 42, 360 - 364 (in German).

Carpenter, M., Frank, J., Winter, D., Peysar, G., 2001. Sampling Duration Effects on Centre of Preassure Summary Measures. Gait \& Posture, 13, $35-40$.

Carter, N.D., Kannus, P, Khan, K. M., 2001. Exercise in the prevention of falls in older people - A systematic literature review examining the rationale and the evidence. Sports Medicine, 31, 427-438.

Corsinovi, L., Bo, M., Ricauda Aimonnino, N., Marinello, R., Gariglio, F., Marchetto, C., Gastaldi, L., Fissore, L., Zanocchi, M., Molaschi, M., 2009. Predictors of falls and hospitalization outcomes in elderly patients admitted to an acute geriatric unit. Archives of Gerontology and Geriatrics, 49, 142 - 145.

Demura, S. , Kitabayaschi, T., 2006. Comparison of power spectrum characteristics of body sway during a static upright standing posture in 
healthy elderly people and young adults. Perceptual and Motor Skills, 102, 467-476.

Doyle, T. L., Newton, R. U., Burnett, A. F., 2005. Reliability of traditional and fractal dimension measures of quiet stance center of pressure in young, healthy people. Arch Phys Med Rehabil, 86, 2034-40.

Gassmann, K.G., Rupprecht, R., Freiberger, E., for the IZG Study Group, 2009. Predictors for occasional and recurrent falls in community-dwelling older people. Zeitschrift für Gerontologie und Geriatrie, 42, 3 - 10.

Horak, F. B., Shupert, C. L., Mirka, A., 1989. Components of postural dysconrtrol in the elderly- a review. Neurobiology of Aging, 10, 727-738.

Johansson, R. \& Magnusson, M., 1991. Human postural dynamics. Critical Reviews in Biomedical Engineering, 18, 413-437.

Kapteyn, T., Bles, W., Njiokiktjien, C., Kodde, L., Massen, C., Mol, J., 1983. Standardization in platform stabilometry being part of posturography. . Agressolgie, 24, 321 - 326.

Karlberg, M., Franson, P., Magnusson, M., 2005. Posturography can be used to screen for primary orthostatic tremor, a rare cause of dizziness. Otology \& Neurology, 26, 1200 - 1203.

Maki, B. E., Holliday, P. J., Topper, A. K., 1994. A prospective-study of postural balance and risk of falling in an ambulatory and independent elderly population. Journals of Gerontology, 49, M72-M84.

Masui, T., Hasegawa, Y., Matsuyama, Y., Sakano, S., Kawasaki, M., Suzuki, S., 2005. Gender differences in platform measures of balance in rural community-dwelling elders. Archives of Gerontology and Geriatrics, 41, 201-209.

Melzer, I., Benjuya, N., Kaplanski, J., 2004. Postural stability in the elderly: a comparison between fallers and non-fallers. Age and Ageing, 33, 602607.

Myklebust, J. B., Lovett, E. G., Myklebust, B. M., Reynolds, N., Milkowski, L., Prieto, T. E., 2009. Two-dimensional coherence for measurement of asymmetry in postural steadiness. Gait \& Posture, 29, 1-5

Myklebust, J. B., Prieto, T. E., Myklebust, B. M., 1995. Evaluation of nonlinear dynamics in postural steadiness time-series. Annals of Biomedical Engineering, 23, 711-719. 
Njiokiktjien, C. \& Folkerts, J., 1971. Deplacement of the body's center of gravity at galvanic stimulation of the labyrinth. Confin Neurol, 33, 46 - 54.

Piirtola, M., Era, P., 2006. Force platform measurements as predictors of falls among older people - A review. Gerontology, 52, 1-16.

Portney, L., Watkins, M., 2000. Foundations of Clinical Research. Applications to Practice. 2nd ed., Prentice Hall Health, Upper Saddle River, NJ, .

Prieto, T. E., Myklebust, J. B., Hoffmann, R. G., Lovett, E. G., Myklebust, B. M., 1996. Measures of postural steadiness: Differences between healthy young and elderly adults. IEEE Transactions on Biomedical Engineering, 43, 956-966.

Prieto, T. E., Myklebust, J. B., Myklebust, B. M., 1993. Characterisation and modeling of postural steadiness in the elderly - a review. IEEE Transactions on Rehabilitation Engineering, 1, 26-34.

Rogind, H., Simonsen, H., Era, P., Bliddal H., 2003. Comparison of Kistler 9861A force platform and Chattecx Balance System (R) for measurement of postural sway: correlation and test-retest reliability. Scandinavian Journal of Medicine \& Science in Sports, 13, 106-114.

Rugeli, D. The effect of functional balance training in frail nursing home residents. Archives of Gerontology and Geriatrics, In Press, Corrected Proof.

Shrout, P., Fleiss, J., 1979. Intraclass Correlations: Uses in Assessing Rater Reliability. Psychological Bulletin 86, 420 - 8.

Soames, R., Atha, J., 1982. The spectral characteristics of postural sway behaviour. Eur J Appl Physiol, 49, 169 - 177.

Tinetti, M., Speechley, M., Ginter, S., 1988. Risk factors for falls among elderly persons living in the community. N Engl J Med, 319, 1701 - 7.

Walter, S., Eliasziw, M., Donner, A., 1998. Sample Size and optimal designs for reliability studies. Stat Med, 17, $101-10$.

Williams, H. G., McClenaghan B. A., Dickerson, J., 1997. Spectral characteristics of postural control in elderly individuals. Archives of Physical Medicine and Rehabilitation, 78, 737-744. 
Table 1

Subjects characteristics

\begin{tabular}{|c|c|c|c|}
\hline & All subjects & Women & Men \\
\hline Number of Subjects (\%) & $30(100 \%)$ & $22(73 \%)$ & $8(27 \%)$ \\
\hline Mean Age ( \pm S.D.) & $77.23( \pm 6.81)$ & $76.01( \pm 7.06)$ & $81.2( \pm 4.43)$ \\
\hline Median Age & 77 & 73 & 82 \\
\hline Age Range & $68-91$ & $68-91$ & $74-86$ \\
\hline
\end{tabular}




\section{Table 2}

Descriptive statistics of the power density in ML direction

\begin{tabular}{lllll}
\hline Variable & Test & Mean \pm S.D. or $\mathrm{n}(\%)$ & Variation & Median \\
\hline Number of subjects & & $30(100 \%)$ & & \\
\multirow{3}{*}{ FFT ML direction } & EO & $159.92( \pm 100.36)$ & 10072.76 & 128.41 \\
& EC & $238.33( \pm 190.96)$ & 36465.91 & 184.22 \\
& NEO & $316.34( \pm 195.42)$ & 38190.13 & 268.46 \\
& NEC & $732.83( \pm 513.93)$ & 264130.53 & 596.82
\end{tabular}

FFT ML direction

Band $1(0-0.5 \mathrm{~Hz})$

$\begin{array}{llll}\text { EO } & 138.01( \pm 92.46) & 8550.22 & 102.65 \\ \text { EC } & 197.29( \pm 176.75) & 31241.37 & 150.43 \\ \text { NEO } & 243.42( \pm 158.71) & 25189.38 & 198.53 \\ \text { NEC } & 526.93( \pm 376.04) & 141408.37 & 416.38\end{array}$

FFT ML direction

Band $2(0.5-2 \mathrm{~Hz})$

$\begin{array}{lllc}\text { EO } & 20.82( \pm 14.36) & 206.43 & 16.71 \\ \text { EC } & 39.03( \pm 26.04) & 678.53 & 34.53 \\ \text { NEO } & 69.74( \pm 61.31) & 3758.89 & 46.96 \\ \text { NEC } & 196.45( \pm 181.93) & 33102.02 & 121.13\end{array}$

FFT ML direction

Band $3(>2 \mathrm{~Hz})$

$\begin{array}{llll}\text { EO } & 1.23( \pm 1.09) & 1.19 & 0.88 \\ \text { EC } & 2.19( \pm 2.08) & 4.35 & 1.37 \\ \text { NEO } & 3.59 \pm( \pm 3.47) & 12.06 & 2.31 \\ \text { NEC } & 10.27( \pm 10.95) & 120.07 & 5.07\end{array}$

Notes: $\mathrm{AO}=$ Normal stand eyes open; $\mathrm{AG}=$ Normal stand eyes closed; NAO = Narrow stand eyes open; NAG = Narrow stand eyes closed; FFT = Fast Fourier Transformations; 
Table 3

Descriptive statistics of the power density in AP direction

\begin{tabular}{lllll}
\hline Variable & Test & Mean \pm S.D. or $\mathrm{n}(\%)$ & Variation & Median \\
\hline Number of Subjects & & $30(100 \%)$ & & \\
\multirow{3}{*}{ FFT AP direction } & EO & $177.95( \pm 112.81)$ & 12728.12 & 158.08 \\
& EC & $348.83( \pm 288.99)$ & 83516.35 & 267.31 \\
& NEO & $164.05( \pm 108.78)$ & 11834.61 & 130.67 \\
& NEC & $497.22( \pm 349.66)$ & 122264.75 & 370.01
\end{tabular}

FFT AP direction

Band $1(0-0.5 \mathrm{~Hz})$

$\begin{array}{llll}\text { EO } & 152.21 \pm 107.81) & 11621.11 & 127.32 \\ \text { EC } & 275.45( \pm 271.55) & 73739.45 & 210.89 \\ \text { NEO } & 127.41( \pm 96.58) & 9328.57 & 93.12 \\ \text { NEC } & 371.88( \pm 266.28) & 70908.19 & 287.57\end{array}$

FFT AP direction

Band $2(0.5-2 \mathrm{~Hz})$

$\begin{array}{llll}\text { EO } & 24.04( \pm 14.75) & 217.81 & 22.53 \\ \text { EC } & 68.39( \pm 59.84) & 3581.78 & 50.08 \\ \text { NEO } & 34.16( \pm 30.78) & 947.87 & 25.71 \\ \text { NEC } & 115.79( \pm 115.66) & 13379.03 & 67.27\end{array}$

FFT AP direction

Band $3(>2 \mathrm{~Hz})$

$\begin{array}{llll}\text { EO } & 1.86( \pm 1.61) & 2.61 & 1.31 \\ \text { EC } & 5.31( \pm 6.74) & 45.55 & 2.74 \\ \text { NEO } & 2.67( \pm 2.55) & 6.53 & 1.88 \\ \text { NEC } & 10.19( \pm 14.49) & 210.23 & 3.48\end{array}$

Notes: EO = Normal stand eyes open; EC = Normal stand eyes closed; NEO = Narrow stand eyes open; NEC = Narrow stand eyes closed;FFT = Fast Fourier Transformations; 


\section{Table 4}

Reliability calculation (complete frequency spectrum, both directions)

\begin{tabular}{lllll}
\hline Test & EO & EC & NEO & NEC \\
\hline Number of Subjects (\%) & $30(100 \%)$ & $30(100 \%)$ & $30(100 \%)$ & $30(100 \%)$
\end{tabular}

Trajectories ML

$\begin{array}{lllll}\text { ICC } & 0.706^{\star} & 0.806^{*} & 0.846^{*} & 0.906^{\star} \\ 99 \% \mathrm{Cl} & 0.349-0.880 & 0.570-0.921 & 0.658-0.937 & 0.791-0.962 \\ \mathrm{CV} & 28 \% & 25 \% & 20 \% & 21 \%\end{array}$

Trajectories AP

\begin{tabular}{lllll} 
ICC & $0.655^{\star}$ & $0.792^{\star}$ & $0.828^{\star}$ & $0.853^{\star}$ \\
$99 \% \mathrm{Cl}$ & $0.258-0.864$ & $0.539-0.914$ & $0.619-0.930$ & $0.675-0.940$ \\
$\mathrm{CV}$ & $24 \%$ & $19 \%$ & $19 \%$ & $26 \%$ \\
\hline
\end{tabular}

* indicates $\mathrm{F}<.001$

Notes: $\mathrm{EO}=$ Normal stand eyes open; $\mathrm{EC}=$ Normal stand eyes closed; NEO $=$ Narrow stand eyes open; NEC = Narrow stand eyes closed; $\mathrm{ML}=$ medio - lateral direction; $\mathrm{AP}=$ anterior posterior direction ICC $=$ Intra Class Correlation Coefficient; $99 \% \mathrm{Cl}=99 \%$ confidence interval $\mathrm{CV}=$ Coefficient of Variation 


\section{Table 5}

Reliability calculation (frequency band $1-3$, both directions)

\begin{tabular}{lllll}
\hline Test & EO & EC & NEO & NEC \\
\hline Number of Subjects & $30(100 \%)$ & $30(100 \%)$ & $30(100 \%)$ & $30(100 \%)$
\end{tabular}

Trajectories band $1 \mathrm{ML}$

ICC

$99 \% \mathrm{Cl}$

$\mathrm{CV}$

Trajectories band $2 \mathrm{ML}$

ICC

$99 \% \mathrm{Cl}$

CV

Trajectories band $3 \mathrm{ML}$

ICC

$99 \% \mathrm{Cl}$

$\mathrm{CV}$

Trajectories band $1 \mathrm{AP}$

ICC

$99 \% \mathrm{Cl}$

CV

Trajectories band 2 AP

ICC

$99 \% \mathrm{Cl}$

CV

Trajectories band 3 AP

ICC

$99 \% \mathrm{Cl}$

CV

$$
0.673^{*}
$$

$0.814^{*}$

$0.814^{*}$

$0.885^{\star}$

$0.275-0.867$

$0.709-0.919$

$0.588-0.924$

$33 \%$

$31 \%$

$24 \%$

$0.744-0.953$

$24 \%$

$\begin{array}{llll}0.802^{*} & 0.772^{\star} & 0.866^{\star} & 0.909^{\star} \\ 0.560-0.919 & 0.495-0.907 & 0.658-0.937 & 0.799-0.963 \\ 27 \% & 22 \% & 21 \% & 22 \%\end{array}$

0.721 *

$0.742^{*}$

$0.808^{*}$

$0.895^{\star}$

$0.381-0.886$

$.426-0.895$

$0.574-0.921$

$0.768-0.957$

$34 \%$

$28 \%$

$22 \%$

$26 \%$

* indicates $\mathrm{F}<.001$

$\begin{array}{llll}0.749^{*} & 0.812^{*} & 0.917^{*} & 0.939^{*}\end{array}$

$0.467-0.902 \quad 0.583-0.923 \quad 0.916-0.966 \quad 0.865-0.975$

$23 \% \quad 20 \% \quad 26 \%$

$\begin{array}{llll}0.865^{*} & 0.746^{*} & 0.889^{*} & 0.930^{*} \\ 0.702-0.942 & 0.437-0.897 & 0.754-0.955 & 0.845-0.971 \\ 26 \% & 25 \% & 26 \% & 20 \%\end{array}$

Notes: $\mathrm{EO}=$ Normal stand eyes open; $\mathrm{EC}=$ Normal stand eyes closed; NEO = Narrow stand eyes open; $\mathrm{NEC}=$ Narrow stand eyes closed; $\mathrm{ML}=$ medio - lateral direction; $\mathrm{AP}=$ anterior posterior direction ICC $=$ Intra Class Correlation Coefficient; $99 \% \mathrm{Cl}=99 \%$ confidence interval $\mathrm{CV}=$ Coefficient of Variation 
Table 6

Fmax test results

\begin{tabular}{lllll}
\hline Variable & complete & band 1 & band 2 & band 3 \\
\hline$F(\max )$ value & 22.22 & 16.56 & 160.51 & 176.61 \\
\hline
\end{tabular}

Notes: Complete: $F$ value for the complete frequency spectrum; Band 1: $F$ value for the low frequency spectrum; Band 2: $F$ value for the intermediate frequency spectrum; Band 3: $F$ value for the high frequency spectrum 
Christoph Bauer, MSc

School of Health Professions

Institute of Physiotherapy

Zurich University of Applied Sciences

Technikumstrasse 71

P.O. Box

8401 Winterthur

Switzerland 